\title{
Immunotherapy Related Adverse Events Predict Treatment Response
}

\author{
Diana Maslov, 2, *, Katharine Thomas ${ }^{1,2,3}$, Victoria Simenson ${ }^{1}$, Caitlin Sullivan ${ }^{2,4}$, \\ Alaa Mohammed ${ }^{5}$, Jessica Boyce ${ }^{2}$, Marc Matrana ${ }^{6}$ \\ ${ }^{1}$ Department of Internal Medicine, Ochsner Clinic Foundation, New Orleans, the United States \\ ${ }^{2}$ Ochsner Clinical School, University of Queensland, New Orleans, the United States \\ ${ }^{3}$ Department of Hematology Oncology, Louisiana State University Health Sciences Center, New Orleans, the United States \\ ${ }^{4}$ Department of Internal Medicine, Louisiana State University Health Sciences Center, New Orleans, the United States \\ ${ }^{5}$ Center for Outcomes and Health Services Research, Ochsner Clinic Foundation, New Orleans, the United States \\ ${ }^{6}$ Department of Hematology/Oncology, Ochsner Cancer Institute, New Orleans, the United States \\ Email address: \\ Diana.maslov@ochsner.org (D. Maslov) \\ ${ }^{*}$ Corresponding author
}

\section{To cite this article:}

Diana Maslov, Katharine Thomas, Victoria Simenson, Caitlin Sullivan, Alaa Mohammed, Jessica Boyce, Marc Matrana. Immunotherapy Related Adverse Events Predict Treatment Response. Journal of Cancer Treatment and Research. Vol. 8, No. 2, 2020, pp. 45-50.

doi: $10.11648 /$ j.jctr.20200802.13

Received: May 17, 2020; Accepted: May 28, 2020; Published: June 9, 2020

\begin{abstract}
Immunotherapy blocks immunoinhibitory pathways and allows for reversal of immunosuppression caused by malignant tumors. Immunotherapy can cause immune-related adverse events (IrAE) including rash, pneumonitis, colitis endocrinopathy, nephritis, adrenal insufficiency, hepatitis, and uveitis. Limited data exist to predict which patients will have the greatest response to therapy and if there is a correlation between IrAE and immunotherapy effectiveness. The aim of this study was to determine the relationship between IrAE and immunotherapy efficacy. A retrospective medical records review was collected of patients with metastatic cancer who received immunotherapy. Data included demographics, Eastern Cooperative Oncology Group Performance Status, imaging, time on treatment, best response, disease progression, and presence or absence of IrAE while on treatment. Treatment response was analyzed using the Response Evaluation Criteria in Solid Tumors guideline, version 1.1. Overall survival probabilities were calculated by the Kaplan-Meier survival method. 456 patients were included for analysis, $175(38.4 \%)$ had an IrAE while on immunotherapy. The development of IrAE correlated with response rate for complete response, partial response, and overall response rate. A significant increase in overall survival was also seen. The presence of IrAE may be a potential predictive indicator for treatment response to immune checkpoint inhibitors.
\end{abstract}

Keywords: Immunotherapy, Adverse Events, Neoplasms, Immunology

\section{Introduction}

Program death-ligand 1 (PD-L1) inhibitors have been developed to promote the activation of antineoplastic $\mathrm{T}$ cells and reduce tumor size [1]. Within lymph nodes, PD-L1 assists in preventing the activation of new cytotoxic $\mathrm{T}$ cells [2]. Within dendritic cells, PD-L1 deactivates cytotoxic T cells. PD-L1 also plays a key role in tumor autophagy, helping cancer cells adjust to the body's environment and continue to proliferate [3]. Inhibitors of PD-L1 allow for tumor-invading cells to be recognized and destroyed by cytotoxic $\mathrm{T}$ cells and prevent tumor autophagy, ultimately stopping malignant cells from adaptation and development [2, 3]. PD-1/PD-L1 inhibitor therapy includes nivolumab, pembrolizumab, atezolizumab, avelumab, and durvalumab. These drugs have been shown to improve outcomes in certain tumor subsets, especially those that harbor a high tumor mutational burden [1]. In one study by Hellmann et al [2] the efficacy of nivolumab \pm ipilimumab in the treatment of non-small cell lung cancer (NSCLC) was enhanced in patients with a high tumor mutational burden compared to patients with less mutations [2]. Immunotherapy has been approved for more than 10 different solid tumor types and for any tumor with high 
levels of microsatellite instability or deficient mismatch repair genes $[4,5]$. Immune checkpoint inhibitors work by blocking immunoinhibitory pathways, allowing reversal of immunosuppression caused by advanced malignant tumor [6]. These immunotherapy drugs can cause immune-related adverse events (IrAE) including rash, pneumonitis, colitis, endocrinopathy (hypo and hyperthryroidism), nephritis, adrenal insufficiency, hepatitis, and uveitis [7, 8]. Although research and knowledge of immune checkpoint inhibitors have increased in the past decade, there is limited data studying the relationship between patient adverse events and immunotherapy efficacy. Due to the lack of research, it is difficult to predict which patients will have the greatest response to therapy. Thus, the aim of this study was to determine the relationship between $\operatorname{IrAE}$ and immunotherapy efficacy.

\section{Materials and Methods}

\subsection{Study Design, Population Setting, and Data Collection}

This retrospective cohort study included patients with metastatic cancer who presented to the Ochsner Cancer Institute between October 1, 2014 and February 1, 2019. Data was gathered through the electronic medical record. Patients who received at least one cycle of PD-1/PD-L1 immunotherapy (nivolumab, pembrolizumab, atezolizumab, or durvalumab) and no other drug in combination during the study period were eligible. Patients who were lost to follow-up and patients who did not receive imaging during their time on immunotherapy and were therefore unable to have Response Evaluation Criteria in Solid Tumors (RECIST) measurements were excluded from the study. This study was approved by the Ochsner Clinic Foundation Institutional Review Board.

\subsection{Study Variables, Data Resources, Primary and Secondary Outcomes}

Demographics (age, sex, race, smoking history), Eastern Cooperative Oncology Group Performance Status at the start of treatment, imaging results (computed tomography [CT] scans, magnetic resonance imaging [MRI], positron emission tomography $[\mathrm{PET}]$ ), time on treatment, best response to treatment, date of progression of disease, and presence or absence of IrAE while on treatment were collected. The primary outcome was the relationship between $\operatorname{IrAE}$ and tumor response to immune-targeted therapy. To determine this relationship, treatment response was analyzed on CT, MRI, or PET imaging with the RECIST version 1.1 criteria [9]. Overall response rate was defined as patients with a partial response or a complete response. Two cohorts of patients were created based on the presence or absence of IrAE. The secondary outcome was overall survival, defined as the time from the first immunotherapy treatment to death or termination of the study (February 1, 2019).

\subsection{Statistical Analysis}

Bivariate analyses were run between IrAE status with patients demographics, medical history, and clinical outcomes. For categorical data, chi-square test was used to compare the groups, and results are reported as frequencies and percentages. For continuous variables, independent sample t-test or Wilcoxon rank sum test was used to compare patient groups. SAS version 9.4 for windows (Cary, NC, USA) was used to conduct all analyses. Tests were performed at a significance level of $\alpha=0.05$. Values were considered statistically significant if $\mathrm{P}$ was $<0.05$.

\subsection{Survival Analysis}

Overall survival probabilities were assessed using Kaplan-Meier methods stratified by IrAE status (present vs absent) over 30 months.

\section{Results}

\subsection{Patient Characteristics}

We identified 456 patients with metastatic cancer who met the study criteria. Their median age was 67.6 years (range from 28-94 years old). The median time on immunotherapy for all patients was 3.7 months. Of the 456 patients included in this study, 175 had an IrAE while on immunotherapy. The patient population was similar between those with IrAE and those without. Demographics were not significantly different between the two groups (Table 1). The majority of patients on immunotherapy had Non Small Cell Lung Cancer (NSCLC), and most patients were on nivolumab or pembrolizumab, which is consistent with the known epidemiology of this disease (Table 2).

Table 1. Demographics.

\begin{tabular}{lll}
\hline & Adverse Events Present & Total \\
\hline Number of Patients & 175 & 456 \\
Male (\%) & $122(69.7)$ & $324(71.1)$ \\
Median Age in years & 67.5 & 67.6 \\
Race: & & \\
Black (\%) & $46(26.3)$ & $135(29.6)$ \\
White (\%) & $124(70.9)$ & $308(67.5)$ \\
Other (\%) & $5(2.9)$ & $13(2.9)$ \\
Smoking History: & & \\
Never/light (\%) & $81(46.3)$ & $206(45.2)$ \\
Heavy (\%) & $94(53.7)$ & $250(54.8)$ \\
Current Smoker (\%) & $22(12.6)$ & $69(15.1)$ \\
ECOG ${ }^{1}$ Performance Status $(\%):$ & $194(42.5)$ \\
0 & $87(49.7)$ & $173(37.9)$ \\
1 & $66(37.7)$ & $64(14.0)$ \\
2 & $18(10.3)$ & $25(5.5)$ \\
3 & $4(2.3)$ & \\
4 & & \\
5 & & \\
\hline
\end{tabular}

${ }^{1}$ ECOG: Eastern Cooperative Oncology Group.

Table 2. Cancer Subtypes and Drug Types.

\begin{tabular}{lll}
\hline & $\begin{array}{l}\text { Adverse Events } \\
\text { Present }\end{array}$ & $\begin{array}{l}\text { Total No. of } \\
\text { Patients }\end{array}$ \\
\hline $\begin{array}{l}\text { No. of Patients } \\
\text { Cancer: }\end{array}$ & 175 & 456 \\
Hepatocellular (\%) & $5(2.9)$ & 20 \\
Melanoma (\%) & $27(15.4)$ & 47 \\
Non Small Cell Lung Cancer (\%) & $74(42.3)$ & 192 \\
\hline
\end{tabular}




\begin{tabular}{lll}
\hline & $\begin{array}{l}\text { Adverse Events } \\
\text { Present }\end{array}$ & $\begin{array}{l}\text { Total No. of } \\
\text { Patients }\end{array}$ \\
\hline Renal Cell (\%) & $23(13.1)$ & 52 \\
Small Cell (\%) & $17(9.7)$ & 40 \\
Urothelial (\%) & $8(4.6)$ & 41 \\
Other (\%) & $21(12)$ & 64 \\
Drug: & & \\
Nivolumab & 102 & 253 \\
Pembrolizumab & 59 & 162 \\
Atezolizumab & 5 & 25 \\
Durvalumab & 9 & 16 \\
\hline
\end{tabular}

Of the 253 patients on nivolumab, $102(40.3 \%)$ had an IrAE. A total of 59 of 162 patients on pembrolizumab, 5 of 25 patients on atezolizumab, and 9 of the 16 patients on durvalumab had an IrAE (Table 2).

Most common IrAE's included hypothyroidism $(n=63,13.8 \%)$, rash $(n=48,10.5 \%)$, and colitis $(n=32,7 \%)$ with adrenal insufficiency, hepatitis, hyperthyroidism, nephritis, hypophysitis, pneumonitis, and uveitis also discussed (Table 3). Most patients with IrAE had NSCLC, followed by Melanoma and Renal Cell Carcinoma (RCC).

Table 3. Type of Immune-related Adverse Event (AE) by Cancer Type.

\begin{tabular}{|c|c|c|c|c|c|c|c|c|}
\hline Immune-Mediated AE & Hepato-cellular & Melanoma & NSCLC & $\begin{array}{l}\text { Renal } \\
\text { Cell } \\
\end{array}$ & $\begin{array}{l}\text { Small } \\
\text { Cell }\end{array}$ & Urothelial & Other & AE Present Total (\%) \\
\hline Hypothyroid & 0 & 11 & 31 & 7 & 7 & 1 & 6 & $63(13.8)$ \\
\hline Rash & 3 & 11 & 20 & 6 & 2 & 2 & 4 & $48(10.5)$ \\
\hline Colitis & 1 & 8 & 10 & 3 & 4 & 1 & 5 & $32(7.0)$ \\
\hline Pneumonitis & 1 & 2 & 17 & 3 & 1 & 1 & 5 & $30(6.6)$ \\
\hline Hepatitis & 1 & 4 & 8 & 3 & 3 & 3 & 1 & $23(5.0)$ \\
\hline Nephritis & 1 & 1 & 10 & 5 & 1 & 1 & 2 & $21(4.6)$ \\
\hline Hyperthyroid & 2 & 0 & 10 & 0 & 1 & 0 & 1 & $14(3.1)$ \\
\hline Adrenal Insufficiency & 0 & 1 & 2 & 3 & 1 & 0 & 0 & $7(1.5)$ \\
\hline Hypophysitis & 0 & 0 & 2 & 0 & 0 & 0 & 1 & $3(.7)$ \\
\hline Uveitis & 0 & 0 & 1 & 0 & 0 & 0 & 0 & $1(.2)$ \\
\hline Adverse Events Total & 9 & 38 & 111 & 30 & 20 & 9 & 25 & \\
\hline
\end{tabular}

\subsection{Therapeutic Response}

In the total population, $108(23.7 \%)$ patients had an overall response (calculated by combining patients with partial and complete responses), and the development of IrAE correlated with response rate (Table 4). Among the patients with a reported IrAE, $63(58.3 \%)$ had an overall response compared to 45 $(41.7 \%)$ of patients without IrAE. Complete response occurred in 38 patients $(8.3 \%)$ overall, $21(55.3 \%)$ of patients with an IrAE and $17(44.7 \%)$ of patients without an IrAE. Partial response occurred in 70 patients $(15.4 \%)$ overall, $42(60.0 \%)$ patients with an IrAE vs $28(40.0 \%)$ patients without an IrAE.

The disease progressed in 217 patients (47.6) overall, and the majority of these patients did not have an IrAE (164 [75.6\%]), Similarly, 131 patients $(28.7 \%)$ had stable disease, and the majority did not have an $\operatorname{IrAE}(72$ [55.0\%]).

\subsection{Survival Analysis}

By study termination, 222 of the 456 patients had died (48.7\%). At 10 months, 103 the 175 patients with IrAE remained on treatment compared to 108 of the 281 patients without IrAE who were still being treated (58.8\% vs $38.4 \%$, $\mathrm{p}<0.001)$. At 24 months, 35 patients of the original 175 patients who had IrAE were continuing treatment, while 26 patients of the original 281 patients without an IrAE were still being treated with immunotherapy (54\% vs $36 \%$ survival probability, $\mathrm{P}<0.001$ ) (Figure 1). The median overall survival had not been reached by the time of analysis. The timing of the onset of IrAEs was not analyzed as IrAE can present many months or even years after immunotherapy treatment has ceased [8].

Figures 2 and 3 show a visual representation of the significance described in Table 4.

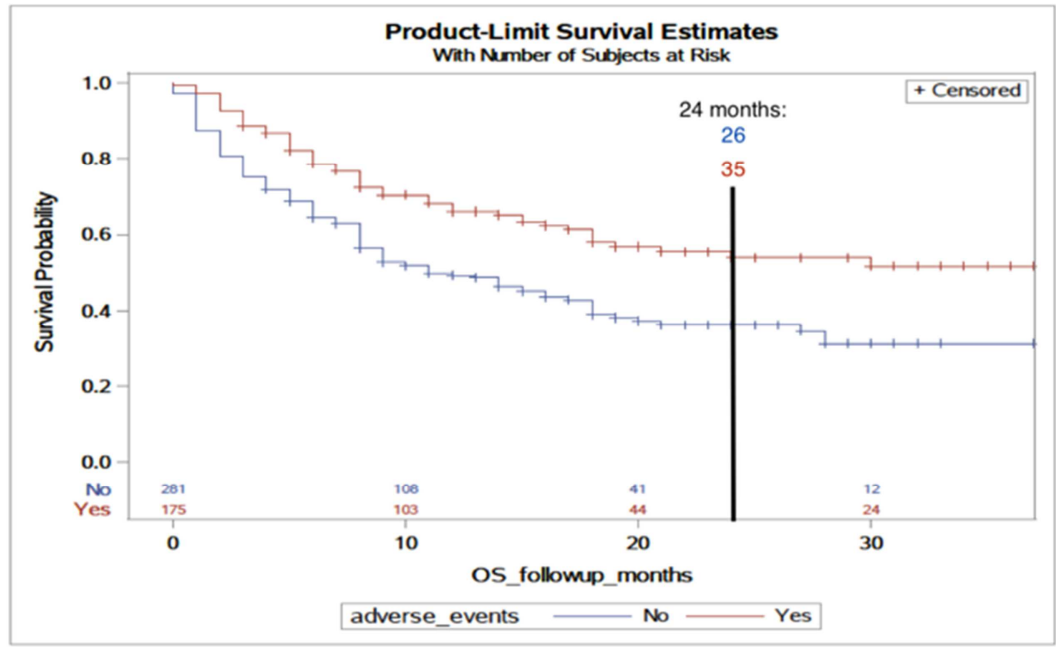

Figure 1. Overall Survival amongst those with and without IrAE while on Immunotherapy 
Table 4. Treatment Response.

\begin{tabular}{llll}
\hline & Adverse Events Absent & Adverse Events Present & Total (\%) \\
\hline No. of Patients & 281 & 175 & 456 \\
Progressive Disease (\%) & $164(75.6)^{\mathrm{a}}$ & $53(24.4)^{\mathrm{a}}$ & $217(47.6)^{\mathrm{b}}$ \\
Stable Disease (\%) & $72(55.0)^{\mathrm{a}}$ & $59(45.0)^{\mathrm{a}}$ & $131(28.7)^{\mathrm{b}}$ \\
Partial Response (\%) & $28(40.0)^{\mathrm{a}}$ & $42(60.0)^{\mathrm{a}}$ & 70 \\
Complete Response (\%) & $17(44.7)^{\mathrm{a}}$ & $21(55.3)^{\mathrm{a}}$ & 38 \\
Overall Response Rate (\%) & $45(41.7)^{\mathrm{a}}$ & $63(58.3)^{\mathrm{a}}$ & $108(23.7)^{\mathrm{b}}$ \\
\hline
\end{tabular}

${ }^{a}$ Percentages for Adverse Events Absent and Present are calculated across the row.

${ }^{b}$ Percentages for Totals are calculated within the column.

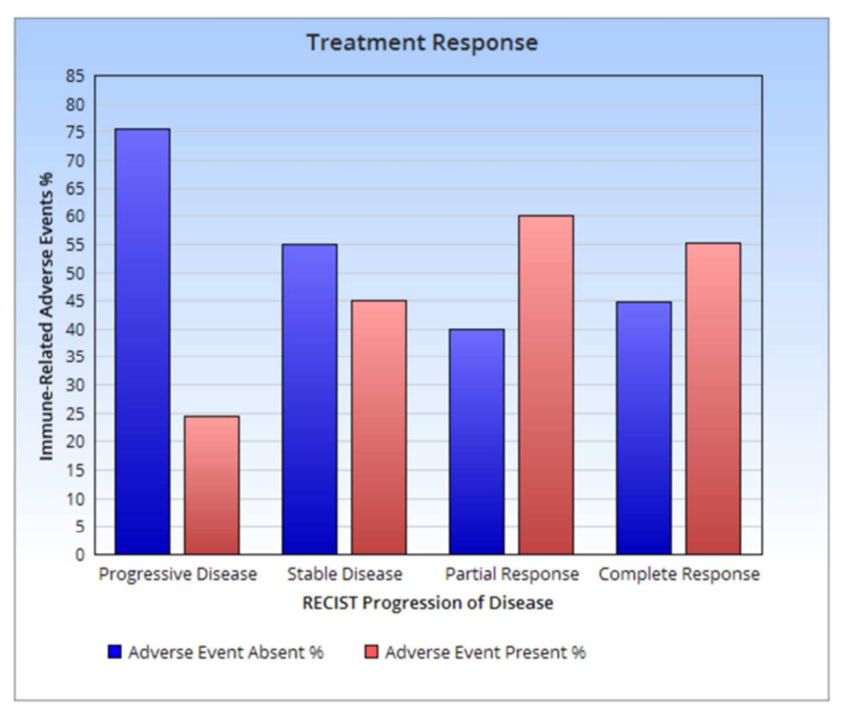

Figure 2. Treatment Response to Immunotherapy according to the Response Evaluation Criteria in Solid Tumors (RECIST) and presence or absence of immune-related adverse events.

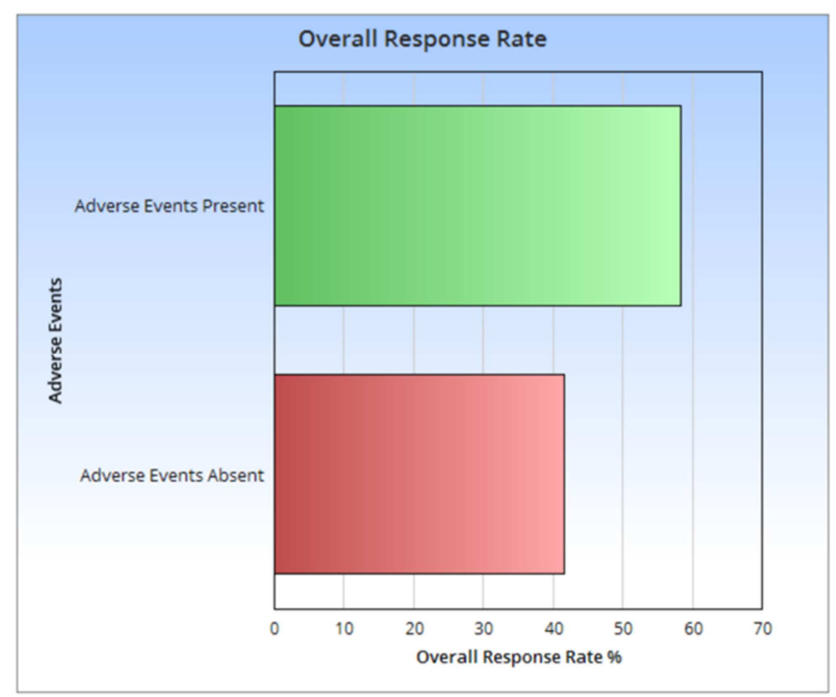

Figure 3. Overall Response Rate and the presence or absence of immune-related adverse events.

\section{Discussion}

IrAEs were present in less than half of our patients on immunotherapy (38.4\%). Hypothyroidism was the most prevalent IrAE in those on immunotherapy, followed by rash. These findings are consistent with the literature, as multiple studies demonstrate that rash, dermatitis, and thyroiditis are the most common adverse events seen in patients on nivolumab or pembrolizumab [9-13]. Interestingly, diarrhea was reported as a prevalent IrAE in a 2017 study conducted by Suarez-Almazor et al. [9]; however, one cannot be certain if diarrhea is specifically immune related [9]. Ramos-Levi et al. [14] investigated 40 patients treated with nivolumab for advanced NSCLC and found that 9 patients had thyroid function changes (22.5\%), further supporting the high incidence of thyroid-related symptoms from immunotherapy treatment [14].

Of note, our study demonstrated that 9 of the 16 of patients on durvalumab had an IrAE, making this drug the most likely to be associated with an IrAE in our population (Table 2). This is of particular interest as our study is the first to document such high prevalence of IrAE. However, the size of the durvalumab treatment group in our study is small, and further investigations are necessary to delve into the relationship between this immunotherapy and IrAE.

Judd et al. [18] reported an association between IrAE and overall response rate in clinical trial patients only (those on pembrolizumab $)(\mathrm{P}=0.007)$ [18].

Our study findings also support that the presence of IrAE while on immunotherapy is associated with prolonged overall survival. Rogardo et al. [15] did not find any significant difference in overall survival in those who were on nivolumab and/or pembrolizumab and those who were not $(\mathrm{P}=0.12)$; however, Rogardo et al. [15] employed a smaller sample size, looking at a smaller variety of cancer types and less immune checkpoint inhibitors compared to our study [14]. A study by Ishihara et al. [12] trends towards an increase in overall survival with nivolumab therapy in patients with IrAE but did not have a significant $\mathrm{p}$ value $(\mathrm{P}=0.072)[12]$. Our study does support a significant trend between the two. Our study indicates a relationship between IrAE and treatment response. Immunotherapy works by activating the immune system to fight cancer cells. IrAE is due to overactivation of certain systems in the body.

Our results showed that the presence of IrAE may represent a potential positive predictive indicator for treatment response to immunotherapy. Our study demonstrated that IrAE were associated with a higher overall response rate (Table 4, Figure 3). Multiple studies have also found an increase in overall response rate in patients on immunotherapy with IrAE vs those without IrAE [15-17]. Lee et al. [16] reported an overall response rate of $65 \%$ for case patients (those who had a case of dermatitis after initiation of PD-1/PD-L1 inhibitor and up to 3 
months after the last dose) and $17 \%$ for controls, $\mathrm{P}=0.0007$ [15]. Sato et al. [17] reported an overall response rate of $63 \%$ for patients with IrAE vs $7.4 \%$ for patients without $(\mathrm{P}<0.01)$ [16].

Although there are many patients that do well on immunotherapy and have no IrAE, by the mechanism of action of immunotherapy increasing a patient's immune response, the therapy should also cause an increase in IrAE. Our study supports this logic. Of course, there are patients who do well on immunotherapy with no IrAE. This study was limited by the retrospective nature of the study and the relative immaturity of the data.

A confounding variable in this study may be that the longer a patient is on treatment, the greater the risk of IrAE. Patients can develop IrAEs for months-years after immunotherapy is discontinued, making this confounder less likely.

\section{Conclusion}

Thus, the aim of this study was to determine the relationship between IrAE and immunotherapy efficacy.

Overall, our results suggest that the presence of IrAE may represent a potential positive predictive indicator for treatment response to immunotherapy. There may be variations in how physicians approach adverse events and continuing therapy. This study supports a more aggressive approach to continuing immunotherapy despite the presence of non-life-threatening IrAE. These adverse events may benefit the patient's overall survival.

Future studies should focus on investigating the benefit of continuing immunotherapy in patients despite moderate to severe symptoms.

\section{Acknowledgements}

Diana Maslov thanks Dr. Jonathan $\mathrm{Lu}$ and Dr. John Kucharczyk for their help with this database. Diana Maslov thanks all authors who contributed. Diana Maslov thanks the Ochsner Post-Doctoral Research Fellowship for the help with this project. Lastly, Diana Maslov thanks Dr. Marc Matrana for his guidance and support.

\section{References}

[1] J. Van den Bulk, E. M. E. Verdegaal, N. F. C. C. de Miranda. "Cancer immunotherapy: broadening the scope of targetable tumours." Open Biol, vol. 8, pp. 6, Jun 2018.

[2] M. D. Hellmann, T. E. Ciuleanu, A. Pluzanski, J. S. Lee, G. A. Otterson, C. Audigier-Valette, E. Minenza, H. Linardou, S. Burgers, P. Salman, H. Borghaei, S. S. Ramalinga. "Nivolumab plus Ipilimumab in Lung Cancer with a High Tumor Mutational Burden," N Engl J Med, vol. 378, pp. 2093-2104, May 2018.

[3] J. M. Michot, C. Bigenwald, S. Champiat, M. Collins, F. Carbonnel, S. Postel-Vinay, A. Bardelou, A. Varga, R. Bahleda, A. Hollebecque, C. Massard, A. Fuerea, V. Ribrag, J. P. Armand, N. Amellal, E. Angevin, N. Noel, C. Boutros, C.
Mateus, C. Robert, J. C. Soria, A. Marabelle, O. Labotte. "Immune-related adverse events with immune checkpoint blockade: A comprehensive review," Eur J, vol. 54, pp. 139-148, Feb 2016.

[4] P. A. Ascierto, R. Addeo, G. Carteni, B. Danieelee, M. De Larentis, G. P. Ianniello, A. Morabito, G. Palmieri, S. Pepe, F. Perrone, S. Pignata, V. Montesarchio. "The role of immunotherapy in solid tumors: report from Campania Society of Oncology Immunotherapy (SCITO) meeting, Naples 2014," J Transl Med, vol. 12, pp. 291, Oct 2014.

[5] M. Robainas, R. Otano, S. Bueno, S. Ait-Oudhia. "Understanding the role of PD-L1/PD1 pathway blockade and autophagy in cancer therapy,” Onco Targets Ther, vol. 10, 1803-1807, Mar 2017.

[6] Mechanism of Action. OPDIVO ${ }^{\circledR}$ (Nivolumab) Restores Active T-Cell Response Directed at the Tumor to Induce an Anti-Tumor Immune Response. Available online: Opdivohcp.com www.opdivohcp.com/advanced-renal-cell-carcinoma/how-opd ivo-works/mechanism-of-action. Accessed 05 May 2019.

[7] Q. Wang, R. Xu. "Immunotherapy-related adverse events (IrAEs): Extraction from FDA drug labels and comparative analysis," JAMIA Open vol. 1, pp. 173-178, April 2019.

[8] M. A. Couey, R. B. Bell, A. A. Patel, M. C. Romba, M. R. Crittenden, B. D. Curti, W. J. Urba, R. S. Leidner. "Delayed immune-related events (DIRE) after discontinuatioin of immunotherapy: diagnostic hazard of autoimmunity at a distance," J. immunotherapy cancer, vol. 7, pp. 165, Jul 2019.

[9] M. E. Suarez-Almazor, S. T. Kim, N. Abdel-Wahab, A. Diab, "Immune-Related Adverse Events With Use of Checkpoint Inhibitors for Immunotherapy of Cancer," Arthritis Rheumatol, vol. 69, issue 4, pp. 687-699, Apr 2017.

[10] J. M. Bourke, M. O'Sullivan, M. A. Khattak. "Management of adverse events related to new cancer immunotherapy (immune checkpoint inhibitors)," Med J Aust, vol. 205, issue 9, pp. 418-424, Nov 2016.

[11] E. A. Eisenhauer, P. Therasse, J. Bogaerts, L. H. Schwartz, D. Sargent, R. Ford, J. Dancey, S. Arbuck, S. Gwyther, M. Mooney, L. Rubinstein, L. Shankar, L. Dodd, R. Kaplan, D. Lacombe, J. Verweij. "New response evaluation criteria in solid tumours: Revised RECIST guideline (version 1.1)," Eur J Cancer, vol. 45, issue 2, pp. 228-247, Jan 2009.

[12] H. Ishihara, T. Takagi, T. Kondo, C. Homma, H. Tachibana, H. Fukuda, K. Yoshida, J. Iizuka, H. Kobayashi, H. Okumi, H. Ishida, K. Tanabe. "Association between immune-related adverse events and prognosis in patients with metastatic renal cell carcinoma treated with nivolumab," Urol Oncol, vol. 37, issue 6, pp. e21-e355. e29.6, Jun, 2019.

[13] T. Fujii, R. R. Colen, M. A. Bilen, K. R. Hess, J. Hajjar, M. E. Suarez-Almazor, A. Alshawa, D. S. Hong, A. Tsimberidou, F. Janku, J. Gong, B. Stephen, V. Subbiah, S. A. Piha-Paul, S. Fu, P. Sharma, T. Mendoza, A. Patel, S. Thirumurthi, A. Sheshadri, F. Meric-Bernstam, A. Naing. "Incidence of immune-related adverse events and its association with treatment outcomes: the MD Anderson Cancer Center experience," Invest New Drugs, vol. 36, issue 4, pp. 638-646, Aug 2018.

[14] A. M. Ramos-Levi, J. Rogado, J. M. Sanchez-Torres, R. Colomer, M. Marazuela. "Nivolumab-induced thyroid dysfunction in patients with lung cancer," Endocrinol Diabetes Nutr, vol. 66, issue 1, pp. 26-34, Jan 2019. 
[15] J. Rogado, J. M. Sanchez-Torres, N. Romero-Laorden, A. I. Ballesteros, V. Pacheco-Barcia, A. Ramos-Levi, R. Arranz, A. Lorenzo, P. Gullon, O. Donnay, M. Adrados, P. Costas, J. Aspa, A. Alfranca, R. Mondejar, R. Colomer. "Immune-related adverse events predict the therapeutic efficacy of ati-PD-1 antibodies in cancer patients," Eur J Cancer, vol. 109, pp. 21-27, Mar 2019.

[16] C. K. M. Lee, S. Li, D. C. Tran, G. A. Zhu, J. Kim, B. Y. Kwong, A. L. S. Chang. "Characterization of dermatitis after PD-1/PD-L1 inhibitor therapy and association with multiple oncologic outcomes: A retrospective case-control study,” J Am Acad Dermatol, vol. 79, issue 6, pp. 1047-1052, Dec 2018.
[17] K. Sato, H. Akamatsu, E. Murakami, S. Sasaki, K. Kanai, A. Hayata, N. Tokudome, K. Akamatsu, Y. Koh, H. Ueda, M. Nakanishi, N. Yamamoto. "Correlation between immune-related adverse events and efficacy in non-small cell lung cancer treatment with nivolumab," Lung Cancer, vol. 115, pp. 71-74, Jan 2018.

[18] J. Judd, M. Zibelman, E. Handorf, J. O’Neill, C. Ramamurthy, S. Bentota, J. Doyle, R. G. Uzzo, J. Bauman, H. Borghaei, E. R. Plimack, R. Mehra, D. M. Geynisman. "Immune-Related Adverse Events as a Biomarker in Non-Melanoma Patients Treated with Programmed Cell Death 1 Inhibitors," Oncologist, vol. 22, issue 10, pp. 1232-1237, Oct 2017. 